\title{
Extrasolar planet detections with gravitational microlensing
}

\section{Shude Mao, Eamonn Kerins and Nicholas J. Rattenbury}

Jodrell Bank Centre for Astrophysics, University of Manchester, Manchester M13 9PL, UK email: (shude.mao, eamonn.kerins, nicholas.rattenbury)@manchester.ac.uk

\begin{abstract}
Microlensing light curves due to single stars are symmetric and typically last for a month. So far about 4000 microlensing events have been discovered in real-time, the vast majority toward the Galactic centre. The presence of planets around the primary lenses induces deviations in the usual light curve which lasts from hours (for an Earth-mass $\left[M_{\oplus}\right]$ planet) to days (for a Jupiter-mass $\left[M_{\mathrm{J}}\right]$ planet). Currently the survey teams, OGLE and MOA, discover and announce microlensing events in real-time, and follow-up teams (together with the survey teams) monitor selected events intensively (usually with high magnification) in order to identify anomalies caused by planets. So far four extrasolar planets have been discovered using the microlensing technique, with half a dozen new planet candidates identified in 2007 (yet to be published). Future possibilities include a network of wide-field $2 \mathrm{~m}$-class telescopes from the ground (which can combine survey and follow-up in the same setup) and a 1m-class survey telescope from space.
\end{abstract}

Keywords. gravitational lensing, Galaxy: centre, planetary systems

\section{Introduction}

Gravitational microlensing refers to the fact that when an intervening object (the lens) is well-aligned with a background star, then the background star will be amplified. Due to relative motions, the source appears brighter as the lens moves closer to the line of sight, and falls back to the baseline as the lens moves away. The resulting light curve is symmetric and follows a characteristic shape. Since microlensing does not depend on whether the lens is luminous or dark, it was first proposed as a way to detect massive compact dark matter object in the Milky Way (Paczynski 1986).

For a source in the Local Group, the chance for a star to be microlensed is small, of the order of $10^{-6}$. To have a realistic yield of microlensing events, very dense fields have to be monitored. The targets so far include the Galactic centre, the Magellanic clouds, and M31. Hundreds of millions of stars are routinely monitored, and thousands of microlensing events have been discovered, with diverse applications (see section 2 and the review by Paczynski 1996).

It was first realised that microlensing can be used to discover extrasolar planets more than 15 years ago (Mao \& Paczynski 1991; Loeb \& Gould 1992). The importance of observing high-magnification events was realised by Griest \& Safizadeh (1998) while the sensitivity of microlensing down to Earth masses was highlighted by Bennett \& Rhie (1996).

It was optimistically written in Mao \& Paczynski (1991) that "A massive search for microlensing of the Galactic bulge stars may lead to a discovery of the first extrasolar planetary systems". In fact, the first discovery by microlensing did not come until 2004 (Bond et al. 2004), while other methods (particularly the radial velocity method, see the contributions by Mayor and Marcy for details) made rapid progress. In the last 
few years, the pace of microlensing discoveries clearly quickened; we expect many more exciting discoveries yet to come.

Two excellent reviews on microlensing detection of extrasolar planets appeared very recently (Rattenbury 2006; Gaudi 2007); the readers are referred to those papers for more in-depth discussions. Future possibilities from the ground and space are discussed in two recent white papers (Gould et al. 2007; Bennett et al. 2007b).

\section{Status of microlensing surveys}

After the proposal by Paczynski (1986), several collaborations started to survey the Galactic centre and the Magellanic clouds (MACHO, Alcock et al. 1993; EROS, Aubourg et al. 1993; OGLE, Udalski et al. 1992; MOA, Muraki et al. 1999). Several groups (e.g. Calchi Novati et al. 2005; de Jong et al. 2006; Kerins et al. 2006) are also observing the M31 where the stellar population is unresolved (Crotts 1992; Gould 1996).

So far, about 4000 events have been discovered real-time, the vast majority towards the Galactic centre; an equal number is likely yet to be identified in the archive. The current discovery rate of microlensing events in real-time by the OGLE $\dagger$ and MOA $\ddagger$ collaborations is close to 1000 events per year (with some overlapping events). The experiment toward M31 can also now issue real-time alerts of microlensing events (Darnley et al. 2007), which may be particularly relevant for the detection of extrasolar planets in M31 (Chung et al. 2006).

The Einstein radius crossing time for a typical event toward the Galactic centre is about 20 days, but can be as short as one day and as long as four years (OGLE-1999BUL-32, Mao et al. 2002). A typical peak magnification is a factor of a few, but events (for bright stars) with peak magnification a few percent above unity have been identified; the highest peak magnification is about 3000 (Dong et al. 2006).

The microlensing surveys have not only detected thousands of microlensing events, they also accumulated photometric and astrometric data for hundreds of millions of stars for over a decade. Unfortunately, much of the database has yet to be fully explored. For example, the data can be used to provide strong constraints on the Galactic structure using the maps of optical depths (Kiraga \& Paczynski 1994), proper motions (e.g. Kozlowski et al. 2006; Rattenbury et al. 2007a), stellar populations (e.g., Stanek et al. 1997; Rattenbury et al. 2007b). However, this potential has yet to be fulfilled partly due to the lack of man-power in the field.

Undoubtedly the most exciting highlight of microlensing in the last few years is the discovery of extrasolar planets from analysis of individual microlensing events, a topic we will turn to next.

\section{Principles of extrasolar planet detection}

A single point lens always creates two images of a background source, one magnified and one de-magnified. One special case is when the source, lens and observer are are all perfectly aligned, then due to axis-symmetry, a ring of images (called Einstein ring) will form (see Fig. 1 for an illustration).

The presence of a planet can perturb the two existing images (and create an extra faint image close to itself). In addition, in some cases, the planet can create an extra pair of bright images when the source is located inside the so-called caustics (see Fig. 1). Thus for caustic-crossing trajectories, much more dramatic deviations in the light curve can be seen. Both types of light curves have been observed in planetary microlensing events.

$\dagger$ see www.astrouw.edu.pl/ $\sim$ ogle/ogle3/ews/ews.html

$\ddagger$ www.phys.canterbury.ac.nz/moa/microlensing_alerts.html 

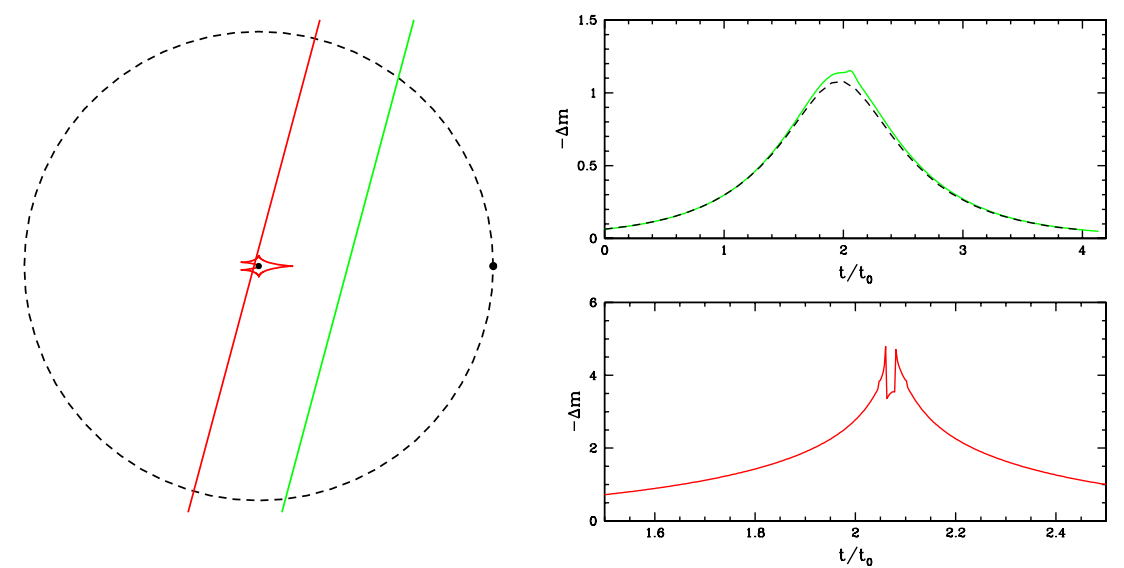

Figure 1. Geometry and light curves of planetary lensing. The primary star is located at the centre of the Einstein ring (dashed curve). A planet of $0.1 \%$ of the mass of the primary star is located to the right on the Einstein ring (indicated by a dot). The central cuspy feature around the primary star is the (central) caustic within which an extra pair of images are created. Two source trajectories are shown, with the corresponding light curves (magnitudes vs. time in units of the Einstein radius crossing time, $t_{0}$ ) shown on the right. The bottom light curve corresponds to the light curve for the trajectory on the left which intercepts the caustic four times. The top light curve corresponds to the trajectory on the right; the dashed curve shows the best fit from the single lens model. The source size is assumed to be $1.67 \times 10^{-3}$ Einstein radius.

Currently the survey teams discover the microlensing events and announce these in real-time. Follow-up teams, together with the survey teams, then observe selected events (typically with high magnification) to identify deviations from the standard single light curve, through either visual inspection (e.g., for $\mu$ Fun $\uparrow$ ) or automated algorithms (Dominik et al. 2007). The latter may allow a better evaluation of the detection efficiency (Cassan 2008). Once an anomaly is detected, a much more intensive observing campaign is launched, involving multiple sites and large international teams. Detailed modelling can then indicate the presence of an extrasolar planet. So far, four extrasolar planets have been discovered (and published) using the gravitational microlensing technique, with a probable six new discoveries made in 2007 (yet to be published).

The rate and duration of the planetary perturbations roughly scale as $q^{1 / 2}$, where $q$ is the mass ratio between the planet and its host star. Typically, planetary deviations last from days for $1 M_{\mathrm{J}}$ planets to hours for one $M_{\oplus}$ planet. It is worth emphasizing that the deviation amplitude can be high even for a $1 M_{\oplus}$ planet, especially when the source crosses the caustics (see Fig. 1). Microlensing is most sensitive to low-mass planets in the so-called 'lensing zone', between 0.6-1.6 Einstein radii (corresponding to roughly 0.5-2.4 $\mathrm{AU}$ in physical units).

Microlensing is also sensitive to free-floating planets, which manifest themselves as short single events lasting hours to days. Furthermore, the method is sensitive to multiple planets (see the contribution from Bennett in these proceedings for an example).

From fitting of a binary light curve, the two most important quantities one can learn about the planet is the mass ratio, $q$, and the (projected) separation in units of the Einstein radius, $b$. If $q \lesssim 10^{-2}$, then the binary companion is likely a planet since typical primary lensing stars have $M \sim 0.3 M_{\odot}$. It is well known that for sparsely sampled data

ๆ http://www.astronomy.ohio-state.edu/ microfun/ 
(e.g., Di Stefano \& Mao 1996, Dominik 1999, Gaudi \& Gould 1997) that different solutions (with distinct mass ratios and separations) can fit the same light curve. However, for a densely sampled light curve, there is often only a unique solution (in terms of $q$ and $b$ ), except for the wide and close binary degeneracy, Dominik 1999). Densely monitored light curves frequently allow one to derive extra physical constraints, for example the angular Einstein radius; in some cases, the lens light can also be seen (e.g., Alcock et al. 2001; Kozlowski et al. 2007). In a few cases cases, the planet mass (not only $q$ ) can be inferred directly (for an illustration, see Bennett's contribution to these proceedings).

\section{What have we learned from microlensing extrasolar planets?}

So far four microlensing extrasolar planets have been published (Bond et al. 2004; Udalski et al. 2005; Beaulieu et al. 2006; Gould et al. 2006). The duration of the planetary deviations lasting from 7 days (for the first case, Bond et al. 2004 ) to about one day, for the lowest mass $\left(\sim 5.5 M_{\oplus}\right)$ extrasolar planet (Beaulieu et al. 2006).

Microlensing clearly probes a different part of the parameter space in the plane of separation vs. planet mass (see Fig. 2 in Bennett et al. 2007b). Compared with other methods (such as the radial velocity method), microlensing can probe lower-mass planets at larger radii, including analogues of the Solar system.

While the statistics of extrasolar planets are still limited, one important conclusion can already be drawn: super-Earths are common (Gould et al. 2006). Considering the modest resources devoted to this method, this is a very important and cost-effective discovery, which is fully consistent with the conclusions from the radial velocity method (see Mayor's contribution in these proceedings) and the planet formation theory (Ida \& Lin 2004).

The lack of detections in high signal-to-noise ratio light curves also allow us to put constraints on the frequency of planets around other stars. Gaudi et al. (2002) studied 43 high-quality light curves (with no planetary signals) and concluded that less than $1 / 3$ of $\sim 0.3 M_{\odot}$ stars have Jupiter-mass companions between 1.5-4 AU (see also Snodgrass et al. 2004), consistent with the planet frequencies around solar-type stars from the radial velocity method.

\section{Future}

The current discovery rate of extrasolar planets from microlensing still lags behind the radial velocity method. To improve the efficiency, one must consider more ambitious strategies, including possibilities from both the ground and space. Theoretically, faster algorithms for searching the best planetary models are also desirable.

Gould et al. (2007) proposed to establish a network of four $2 \mathrm{~m}$ telescopes (or eight $1.3 \mathrm{~m}$ telescopes for the same photon gathering power) strategically located across the globe, with the primary goal of searching for Earth-mass planets. Ideally, each telescope would have 4 square degrees of view, with a cadence of about 10 minutes. Such a network would monitor 4 fields with a total of 16 square degrees continuously. The main advantage of such a network is that it combines the survey and follow-up teams in the same setup, thus avoiding the division between survey and follow-up networks. The upgraded MOA telescope (MOA-II) has a field of view of about 2 square degrees. This large field of view already combines survey and follow-up to some degree, which enabled the collaboration to make several important discoveries in 2007 (see the contribution by Sumi in these proceedings). Gould et al. (2007) showed that if each host star has two identical mass planets between 0.4-20 AU (uniformly distributed in per decade of separation) an aggressive wide-field survey can detect half a dozen $1 M_{\oplus}$ planets each year. The MOA-II and the soon-to-be-upgraded OGLE (OGLE-IV) can already be regarded as part of such 
a network. However, to complete the whole network, more funding is still needed. Efforts are currently under way to secure funding from various agencies.

Bennett et al. (2007b) proposed the Microlensing Planet Finder satellite mission to search for extrasolar planets. This will put a $1.1 \mathrm{~m}$ telescope in space, equipped with a CCD camera with 0.7 sq. degree of field of view. The advantage is that there are no atmospheric effects and the spatial resolution is much higher, allowing fainter stars to be probed. Since the lower limit of the extrasolar mass we can detect is determined by the finite source size (which smooths and reduces the magnification), a space satellite can detect lower mass planets. Furthermore, the lens light can be routinely detected from space Bennett et al. (2007a), which, combined with other information, allows us to not only detect but also characterize extrasolar planets from $0.1 M_{\oplus}$ upwards in mass and ranging from $0.5 \mathrm{AU}$ to essentially infinity in separation. The cost of the mission is not cheap, about $\$ 390$ million, but the reward is also substantially higher. Its capability very nicely complements the Kepler mission in the discovery space.

Binary/planetary light curves are diverse. For an observed light curve, it is non-trivial to find the best-fit model and explore the parameter space fully. There are two difficult issues that make the exercise non-trivial; both are related to the presence of caustics. When a point source sits on a caustic, in principle its magnification is singular (infinite under geometric optics). However, stars have finite sizes and thus their microlensed magnification is always finite. To obtain the precise value, one must integrate over the singularity. Several methods (Gould \& Gaucherel 1997; Dominik 2007; including ray-shooting techniques, see Bennett \& Rhie 1996, Rattenbury et al. 2002) have been proposed, but it remains to be seen whether there are better ways of doing this. The second difficulty is that the $\chi^{2}$ surface is not smooth, particularly for caustic-crossing events. Most optimization routines cannot easily jump over the sharp features to find the best solution. The problems are tractable for a single planet system, but become much more challenging for multiple planets. A completely satisfactory solution has yet to be found.

\section{Summary}

The progress in the last few years clearly demonstrates that the microlensing method can be used to discover extrasolar planets. The method is based on General Relativity and simple geometry. So far, about ten extrasolar planets have been discovered. These extrasolar planets are further away from the host stars than those discovered by the radial velocity method, and so it probes a different part of parameter space. The method will provide important statistical information about extrasolar planets, e.g., the microlensing planets already allow us to draw the remarkable conclusion that super-Earths are quite common around stars (Gould et al. 2006). A drawback is that microlensing is a one-time observation and most of the detected planets are at a distance of several kpc and thus too far away for direct imaging follow-up.

With more funding, microlensing has the possibility to make ground-breaking discoveries about Earth-mass planets from both the ground and space. The method complements other methods quite well in the discovery space.

\section{Acknowledgements}

SM thanks the local organisers, in particular Prof. Ji-lin Zhou, for travel support and a stimulating meeting at the ancient Chinese city of Suzhou.

\section{References}

Alcock, C., et al. 1993, Nature, 365, 621 
Alcock, C., et al. 2001, Nature, 414, 617

Aubourg, E., et al. 1993, Nature, 365, 623

Beaulieu, J. -P., et al. 2006, Nature, 439, 437

Bennett, A. \& Rhie, S. H. 1992, ApJ, 472, 660

Bennett, D. P., Anderson, J., \& Gaudi, B. S. 2007a, ApJ, 660, 781

Bennett, D. P., et al. 2007b, arXiv:0704.0454

Bond, I. A., et al. 2004, ApJ, 606, L155

Calchi Novati, S. et al. 2005, A\& A, 443, 911

Cassan, A., Sumi, T., \& Kubas, D. 2008, in: Y.-S. Sun, S. Ferraz-Mello \& J.-L. Zhou, (eds.), Exoplanets: Detection, Formation and Dynamics, Proc. IAU Symposium No. 249 (Suzhou,China), p. 31

Chung, S. -J., Kim D., Darnley, M. J., Duke, J. P., Gould, A., Han, C., Jeon, Y. -B., Kerins, E., Newsam, A., \& Park, B. -G. 2006, ApJ, 650, 432

Crotts, A. P. S. 1992, ApJ, 399, 43

Darnley, M. J., Kerins, E., Newsam, A., Duke, J. P., Goud, A., Han, C., Ibrahimov, M. A., Im, M., Jeon, Y. -B., Karimov, R. G., Lee, C. -U., \& Park, B. -G. 2007, ApJ, 661, L45

Di Stefano, R., Mao, S. 1996, ApJ, 457, 93

Dominik, M. 1999, A\&A, 349, 108

Dominik, M. 2007, MNRAS, 377, 1679

Dominik, M., Rattenbury, N. J., Allan, A., Mao, S. et al., 2007 MNRAS, 380, 792

Dong, S., DePoy, D. L., Gaudi, B. S., Gould, A. et al. 2006, ApJ, 642, 842

Gaudi, B. S. \& Gould, A. 1997, ApJ, 486, 85

Gaudi, B. S., et al. 2002, ApJ, 566, 463

Gaudi, B. S. 2007, in: D. Fischer, F. Rasio, S. Thorsett and A. Wolszczan (eds.), Extreme Solar Systems (ASP Conference Series), arXiv:0711.1614

Gould, A. 1996, ApJ, 470, 201

Gould, A. \& Loeb, A. 1992, ApJ, 396, 104

Gould A. \& Gaucherel C. 1997, ApJ, 477, 580

Gould, A., et al. 2006, ApJ, 644, L37

Gould, A., Gaudi, B. S., \& Bennett, D. P 2007, arXiv:0704.0767

Griest, K. \& Safizadeh, N. 1998, ApJ, 500, 37

Ida, S. \& Lin, D. N. C. 2004, ApJ, 616, 567

de Jong, J. et al. 2006, A\&SA, 446, 855

Kerins, E. 2006, MNRAS, 365, 1099

Kiraga, M. \& Paczynski, B. 1994, ApJ, 430, L101

Kozlowski, S., Wozniak, P. R., Mao, S., Smith, M. C., Sumi, T., Vestrand, W. T., \& Wyrzykowski, L. 2006, MNRAS, 370, 435

Kozlowski, S., Wozniak, P. R., Mao, S., \& Wood, A. 2007, ApJ, in press

Mao, S., Smith, M. C., Wozniak, P., Udalski, A., Szymanski, M., Kubiak, M., Pietrzyski, G., Soszyski, I., \& Zebrun, K. 2002, MNRAS, 329, 349

Mao, S. \& Paczynski, B. 1991, ApJ, 374, L37

Muraki, Y., Sumi, T., Abe, F., Bond, I. et al. 1999, Progress of Theoretical Physics, 133, 233

Paczynski, B. 1986 ApJ, 304, 1

Paczynski, B. 1996 ARAA, 34, 419

Rattenbury, N. J., Bond, I. A., Skuljan, J., \& Yock, P. C. M. 2002, MNRAS, 335, 159

Rattenbury, N. J. 2006, Modern Physics Letters A, 21, 919

Rattenbury, N. J., Mao, S. Debattista, V. P., Sumi, T., Gerhard, O., \& de Lorenzi, F. 2007, $M N R A S, 378,1165$

Rattenbury, N. J., Mao, S., Sumi, T., \& Smith, M. C. 2007 MNRAS, 378, 1064

Snodgrass, C., Horne, K., \& Tsapras, Y. 2004, MNRAS, 351, 967

Stanek, K. Z., Udalski, A., Szymanski, M., Kaluzny, J., Kubiak, M., Mateo, M., \& Krzeminski, W. 1997, ApJ, 477, 163

Udalski, A., Szymański, M., Kałużny, J., Kubiak, M., \& Mateo, M. 1992, Acta Astronomica, 42, 253

Udalski, A., et al. 2005, ApJ, 628, L109 